\title{
GRB 050509B: CONSTRAINTS ON SHORT GAMMA-RAY BURST MODELS ${ }^{1}$
}

\author{
J. Huorth ${ }^{2}$ J. Sollerman, ${ }^{2,3}$ J. Gorosabel ${ }^{4}$ J. Granot ${ }^{5}$ S. Klose, ${ }^{6}$ C. Kouveliotou, ${ }^{7}$ J. Melinder, ${ }^{3}$ E. Ramirez-Ruiz, ${ }^{8,9}$ \\ R. Starling, ${ }^{10}$ B. Thomsen, ${ }^{11}$ M. I. Andersen, ${ }^{12}$ J. P. U. Fynbo, ${ }^{2}$ B. L. Jensen, ${ }^{2}$ P. M. Vreeswijk, ${ }^{13}$ J. M. Castro Cerón, ${ }^{2}$ \\ P. Jakobsson, ${ }^{2}$ A. Levan, ${ }^{14}$ K. Pedersen, ${ }^{2}$ J. E. Rhoads, ${ }^{15}$ N. R. Tanvir, ${ }^{16}$ D. Watson, ${ }^{2}$ And R. A. M. J. WiJers ${ }^{10}$ \\ Received 2005 June 6; accepted 2005 August 1; published 2005 August 18
}

\begin{abstract}
We have obtained deep optical images with the Very Large Telescope at ESO of the first well-localized shortduration gamma-ray burst, GRB 050509B. From $V$ and $R$ imaging, initiated $\sim 2$ days after the GRB trigger and lasting up to three weeks, we detect no variable object inside the small Swift XRT X-ray error circle down to $2 \sigma$ limits of $V=26.5$ and $R=25.1$. The X-ray error circle includes a giant elliptical galaxy at $z=0.225$, which has been proposed as the likely host of this GRB. Our limits indicate that if the GRB originated at $z=0.225$, any supernova-like event accompanying the GRB would have to be over 100 times fainter than normal Type Ia SNe or Type Ic hypernovae, 5 times fainter than the faintest known Ia or Ic SNe, and fainter than the faintest known Type II SNe. Moreover, we use the optical limits to constrain the energetics of the GRB outflow. Simple models indicate that unless the intrinsic energy in the outflow from GRB $050509 \mathrm{~B}$ was $\ll 10^{51}$ ergs, there was very little radioactive material with efficient decay timescales for generating a large luminosity. These limits strongly constrain progenitor models for this short GRB.
\end{abstract}

Subject headings: gamma rays: bursts - supernovae: general

\section{INTRODUCTION}

While it is now well established that long-duration $\gamma$-ray bursts (GRBs) coincide with the explosions of massive stars leading to very energetic core-collapse supernovae (SNe; Galama et al. 1998; MacFadyen \& Woosley 1999; Bloom et al. 1999; Stanek et al. 2003; Hjorth et al. 2003), the origin of short/hard GRBs, characterized as having durations $<2 \mathrm{~s}$ and hard spectra (Kouveliotou et al. 1993), remains unknown. There

\footnotetext{
Based on observations collected at the European Southern Observatory, Paranal, Chile (ESO program 075.D-0261).

${ }^{2}$ Dark Cosmology Centre, Niels Bohr Institute, University of Copenhagen, Juliane Maries Vej 30, DK-2100 Copenhagen Ø, Denmark; jens@astro.ku.dk, jfynbo@astro.ku.dk, brian_j@astro.ku.dk, josemari@alumni.nd.edu, pallja@ astro.ku.dk, kp@astro.ku.dk, darach@astro.ku.dk.

${ }^{3}$ Department of Astronomy, Stockholm University, AlbaNova, S-106 91 Stockholm, Sweden; jesper@astro.su.se, jens@astro.su.se.

${ }^{4}$ Instituto de Astrofísica de Andalucía, CSIC, P.O. Box 03004, E-18080 Granada, Spain; jgu@iaa.es.

${ }^{5}$ Kavli Institute for Particle Astrophysics and Cosmology, Stanford University, P.O. Box 20450, Mail Stop 29, Stanford, CA 94309; granot@slac .stanford.edu.

${ }^{6}$ Thüringer Landessternwarte Tautenburg, Sternwarte 5, D-07778 Tautenburg, Germany; klose@tls-tautenburg.de.

${ }^{7}$ NASA Marshall Space Flight Center, National Space Science Technology Center, XD-12, 320 Sparkman Drive, Huntsville, AL 35805; chryssa.kouveliotou-1@ nasa.gov.

${ }^{8}$ Institute for Advanced Study, Einstein Drive, Princeton, NJ 08540; enrico@ias.edu.

${ }^{9}$ Chandra Fellow.

${ }^{10}$ Astronomical Institute "Anton Pannekoek," University of Amsterdam, NL-1098 SJ Amsterdam, Netherlands; starling@science.uva.nl, rwijers@ science.uva.nl.

${ }^{11}$ Department of Physics and Astronomy, University of Aarhus, DK-8000 Århus C, Denmark; bt@phys.au.dk.

${ }^{12}$ Astrophysikalisches Institut Potsdam, An der Sternwarte 16, D-14482 Potsdam, Germany; mandersen@aip.de.

${ }^{13}$ European Southern Observatory, Alonso de Córdova 3107, Casilla 19001, Santiago 19, Chile; pvreeswi@eso.org.

${ }^{14}$ Department of Physics and Astronomy, University of Leicester, University Road, Leicester LE1 7RH, UK; anl@star.le.ac.uk.

${ }^{15}$ Space Telescope Science Institute, 3700 San Martin Drive, Baltimore, MD 21218; rhoads@stsci.edu.

${ }^{16}$ Centre for Astrophysics Research, University of Hertfordshire, College Lane, Hatfield AL10 9AB, UK; nrt@star.herts.ac.uk.
}

have been as yet no afterglow detections in the very few cases where searches for optical counterparts of short GRBs have been performed, primarily due to the lack of early and precise localizations (Kehoe et al. 2001; Gorosabel et al. 2002; Hurley et al. 2002; Klotz et al. 2003).

Recently, the Swift satellite (Gehrels et al. 2004) provided the first rapid and accurate X-ray localization of a short/hard GRB, opening the window for rapid progress on the origin of short GRBs. GRB 050509B (Gehrels et al. 2005) was detected on 2005 May 9 at 04:00:19.23 (UT) by the Swift Burst Alert Telescope. It was a short $(40 \mathrm{~ms})$ and fairly hard burst. The Swift X-Ray Telescope (XRT) determined the source location to be R.A. $=12^{\mathrm{h}} 36^{\mathrm{m}} 13.58$, decl. $=+28^{\circ} 59^{\prime} 01^{\prime \prime} 3$ (J2000.0, error radius 9".3; Gehrels et al. 2005).

The error region of GRB 050509B was observed by several groups (see, e.g., Bloom et al. 2005; Gehrels et al. 2005; Cenko et al. 2005; Castro-Tirado et al. 2005). Remarkably, the burst error circle overlaps with a giant elliptical galaxy, 2MASX J12361286+2858580 (hereafter G1) at $z=0.225$ (see Fig. 1 and Bloom et al. 2005), belonging to the cluster of galaxies NSC $\mathrm{J} 123610+285901$ (Gal et al. 2003). The probability of a chance alignment of a GRB and such a galaxy is of order $\sim 10^{-3}$. Assuming that G1 is therefore the host galaxy, Bloom et al. (2005) and Gehrels et al. (2005) argue that the likely origin of GRB 050509B is a NS-NS (neutron star) or NS-BH (black hole) merger.

It should be noted that a merger does not necessarily imply the absence of optical or other long-wavelength phenomena after the GRB. For example, the "mini-SN" model (Li \& Paczyński 1998) predicts a bright optical flash of much shorter duration than the one from a "normal" SN, typically of about 1 day. But there are alternative scenarios for the origin of short GRBs. Zhang et al. (2003) have suggested that short GRBs may be a variant of long GRBs, e.g., collapsar-like events leading to stripped-core, core-collapse $\mathrm{SNe}$, much like those seen in conjunction with long GRB afterglows (see also Ghirlanda et al. 2004; Yamazaki et al. 2004). An alternative suggestion is that short GRBs are related to thermonuclear explosions, leading to Type Ia SNe (Dar \& De Rújula 2004; Dado et al. 2005). Finally, 

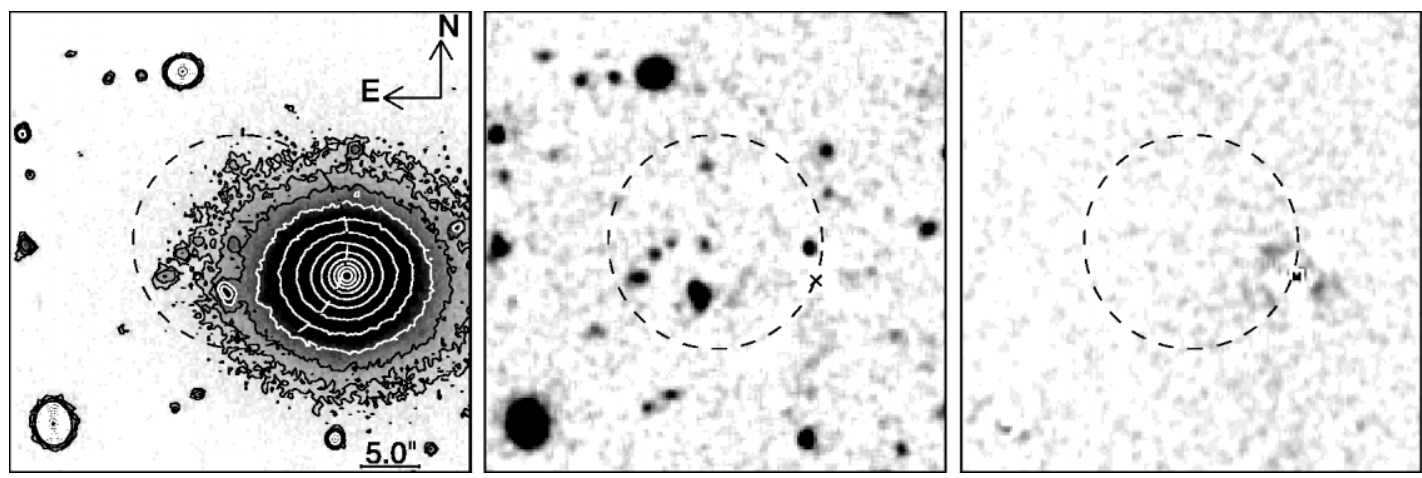

FIG. 1.-Left: First-epoch $V$ image (3.92 days after burst) showing the putative host elliptical galaxy G1 and several faint galaxies in the XRT error circle. Middle: Same, after subtraction of a fit to G1. The cross marks the location of the center of G1. North of it is the new detected source, which may be a foreground or background source or a companion to G1. Right: Difference between last-epoch (22.86 days after burst) and first-epoch $V$ images.

Germany et al. (2000) even suggested that the peculiar Type II SN 1997cy was related to the short GRB 970514 based on their temporal and spatial coincidence. It is obvious from the above that a search for a SN associated with GRB 050509B would help constrain both the energetics of short GRBs and, possibly, their progenitor models (Fan et al. 2005).

We have, therefore, obtained deep images of the XRT error circle at the expected peak time of the putative $\mathrm{SN}$, as well as early images for comparison. In this Letter we present our observations and analysis $(\S 2)$ and discuss the constraints these set on short GRB energetics and progenitor models ( $\S 3$ ). A cosmology with $H_{0}=70 \mathrm{~km} \mathrm{~s}^{-1} \mathrm{Mpc}^{-1}, \Omega_{m}=0.3$, and $\Omega_{\Lambda}=0.7$ is assumed throughout this Letter.

\section{OBSERVATIONS AND DATA ANALYSIS}

We obtained deep $V$ - and $R$-band images containing the XRT error circle with the FORS instruments on the Kueyen and Antu $8.2 \mathrm{~m}$ unit telescopes of the ESO Very Large Telescope at several epochs (Table 1). The data obtained 8 and 12 days after burst were strongly affected by the proximity of the Moon. Consequently, our deepest images were obtained during the first and last sets of observations (a few days and $\sim 3$ weeks after burst).

The data were bias-subtracted, overscan-corrected, and flatfielded using morning-sky flats. The photometric calibration was based on known FORS2 zero points and verified against the SDSS and photometry of the field obtained at the Tautenburg observatory. We estimated the field limiting magnitudes by doing photometry on a large $(\sim 50)$ number of objects. We used the IRAF task phot with an aperture of twice the seeing disk, and obtained the $3 \sigma$ limiting magnitudes given in Table 1 from the errors on the derived magnitudes.

The first image, obtained 1.85 days after the burst, revealed a large number of very faint objects inside the XRT error circle, as well as G1 (see Fig. 1 and Hjorth et al. 2005; Gehrels et al. 2005; Bloom et al. 2005). The surface brightness of G1 within the error circle is, however, only at a level of $20 \%$ of the night-sky level in both the $V$ and the $R$ bands, with the exception of the central 1 " of G1, corresponding to less than $1 \%$ of the error circle. The pixel-to-pixel photon noise is therefore only marginally affected by $\mathrm{G} 1$.

To search for variable objects we subtracted the early images from the late images in each band. The images were aligned, the sky background subtracted, and the images scaled to the same brightness level. We also convolved the image with the best seeing with a spatially variable kernel to match the inferior seeing of the other image, according to the method outlined in Alard \& Lupton (1998). This provided very clean subtractions, except for near the center of the galaxy (Fig. 1). No variable sources were detected.

The elliptical galaxy G1 has a simple morphology. It is therefore possible to fit and subtract a smooth model. This allows detection of superposed point sources with a pixel-to-pixel noise that is reduced by a factor $\sqrt{2}$, formally corresponding to a $0.38 \mathrm{mag}$ deeper limit. A smooth fit of G1 was established by dividing the area around the galaxy into annuli of increasing widths, centered on the nucleus of the galaxy. A robust fitting technique was used to fit a harmonic series to pixel values within each of the annuli (Sodemann \& Thomsen 1994). The full model was obtained by cubic spline interpolation between the harmonic coefficients in the radial direction. Finally, the smooth model was subtracted from the galaxy image (Fig. 1). In the subtracted image we only detect one new faint $(V \sim 26)$ object inside the XRT error circle, 0.78 east and 2".56 north of the G1 galaxy center. This object does not appear to be variable. No other object brighter than the already known sources (Bloom et al. 2005) is present inside the XRT error circle.

To determine the detection limit in the images, a point-spread function was constructed from stars in the field. By superposing

TABLE 1

LOG OF OBSERVATIONS

\begin{tabular}{cccccc}
\hline \hline $\begin{array}{c}\text { Date } \\
(\mathrm{UT})\end{array}$ & $\begin{array}{c}\text { Phase } \\
\text { (days past GRB) }\end{array}$ & Band & $\begin{array}{c}\text { Exposure Time } \\
(\mathrm{s})\end{array}$ & $\begin{array}{c}\text { Seeing } \\
(\operatorname{arcsec})\end{array}$ & Limiting Magnitude \\
\hline 2005 May 11.02 $\ldots \ldots$. & 1.85 & $R$ & 2700 & 0.9 & 26.6 \\
2005 May 13.09 $\ldots \ldots$. & 3.92 & $V$ & 2700 & 0.9 & 27.5 \\
2005 May 17.10 $\ldots \ldots$. & 7.93 & $V$ & 1800 & 0.7 & 25.0 \\
2005 May 22.05 $\ldots \ldots$. & 11.88 & $V$ & 1800 & 1.0 & 24.2 \\
2005 Jun 1.00 $\ldots \ldots \ldots$ & 22.83 & $R$ & 2700 & 0.9 & 26.7 \\
2005 Jun 1.03 $\ldots \ldots \ldots$ & 22.86 & $V$ & 2700 & 1.0 & 27.5 \\
\hline
\end{tabular}

Note. - The quoted $3 \sigma$ limiting magnitudes are measured in the field in a $2 \times$ FWHM aperture. The limiting magnitudes become progressively smaller toward the center of G1. 
artificial stars on $\mathrm{G1}$, we find that stars of $V \simeq 26.5$ and $R \simeq$ 25.1 are recovered in the difference images with $95 \%$ confidence, except within the central 1 " of the galaxy core. In the very nucleus of G1, the galaxy subtraction is poor and we can only detect a source of $V \sim 24$. The corresponding limits for the galaxy subtracted images are 0.3-0.4 mag deeper.

\section{DISCUSSION}

We plot the limits derived in $\S 2$ in Figure 2 along with a number of SN light curves as they would appear at $z=0.225$. The Type Ia templates are from Nugent's compilation ${ }^{17}$ and include (1) a template of a normal Type Ia SN (Nugent et al. 2002) and (2) a template based on the very subluminous Type Ia supernovae SN 1991bg and SN 1999by. The Type Ic SNe plotted are (3) the very energetic Type Ic SN 1998bw associated with the long GRB 980425 (Galama et al. 1998) and (4) the faint, fast-rise Type Ic SN 1994I (Richmond et al. 1996), which was not associated with a GRB but provides a good fit to the light-curve bump in XRF 030723 (Fynbo et al. 2004). Figure 2 clearly demonstrates that even the faintest of these $\mathrm{SNe}$ would have been detected at the time of our observation at a level $\sim 1.8$ mag brighter than our limit $(>5.2 \mathrm{mag}$ fainter than a SN like SN 1998bw).

Type II SNe come in various flavors, the faintest of which are Type IIP. Our limit of $V=26.5$ translates into $M_{B}>-13.3$ at $z=0.225$. All the SN peak magnitudes included in Richardson et al. (2002) are brighter than this, including the faintest Type IIP $\mathrm{SNe}$.

From the above we conclude that if GRB 050509B was associated with a normal bright $\mathrm{SN}$, either its host galaxy must be at a high redshift $(z \gtrsim 1.2)$, consistent with the constraints on the redshifts of the faint galaxies in the XRT error circle (see Bloom et al. 2005), or, if it indeed is at $z=0.225$, its SN light must have been extinguished by dust along the line of sight. The latter option appears unlikely as G1 is an elliptical galaxy with very little star formation (Bloom et al. 2005; Gehrels et al. 2005) and the likely background sources do not appear strongly reddened. We can therefore conclude that there was no SN of known type and characteristics associated with GRB 050509B if it occurred in G1. There remains a (small) probability that GRB 050509B was at a much higher redshift. However, in the following we proceed under the assumption that $\mathrm{G} 1$ is the host galaxy.

The absence of a SN rules out models predicting a normal SN Ia associated with short GRBs (Dar \& De Rújula 2004; Dado et al. 2005). Likewise, our observations disfavor a GRB 050509B progenitor similar to long GRBs, i.e., a collapsar origin. Observations of long GRBs at $z<0.7$ are consistent with all having SN bumps (Zeh et al. 2004), and all GRBs at $z<0.4$ have had SN features (GRB 980425, $z=0.0085$ [Galama et al. 1998]; GRB 031203, $z=0.1055$ [Malesani et al. 2004]; GRB 030329, $z=0.1685$ [Hjorth et al. 2003]; GRB $011121, z=0.362$ [Garnavich et al. 2003]). The situation is less clear regarding X-ray flashes (XRFs); a bright $\mathrm{SN}$ was associated with XRF $020903(z=0.251)$, but no SN (and no optical afterglow) was detected in XRF 040701 (with a probable $z=0.215$ ) down to a limit at least 3 mag fainter than SN 1998bw (Soderberg et al. 2005).

We now proceed to use our derived limits to constrain the energetic properties of the outflow from GRB 050509B. Bloom et al. (2005) find that both the isotropic equivalent energy

\footnotetext{
${ }^{17}$ See http://supernova.lbl.gov/ nugent/nugent_templates.html.
}

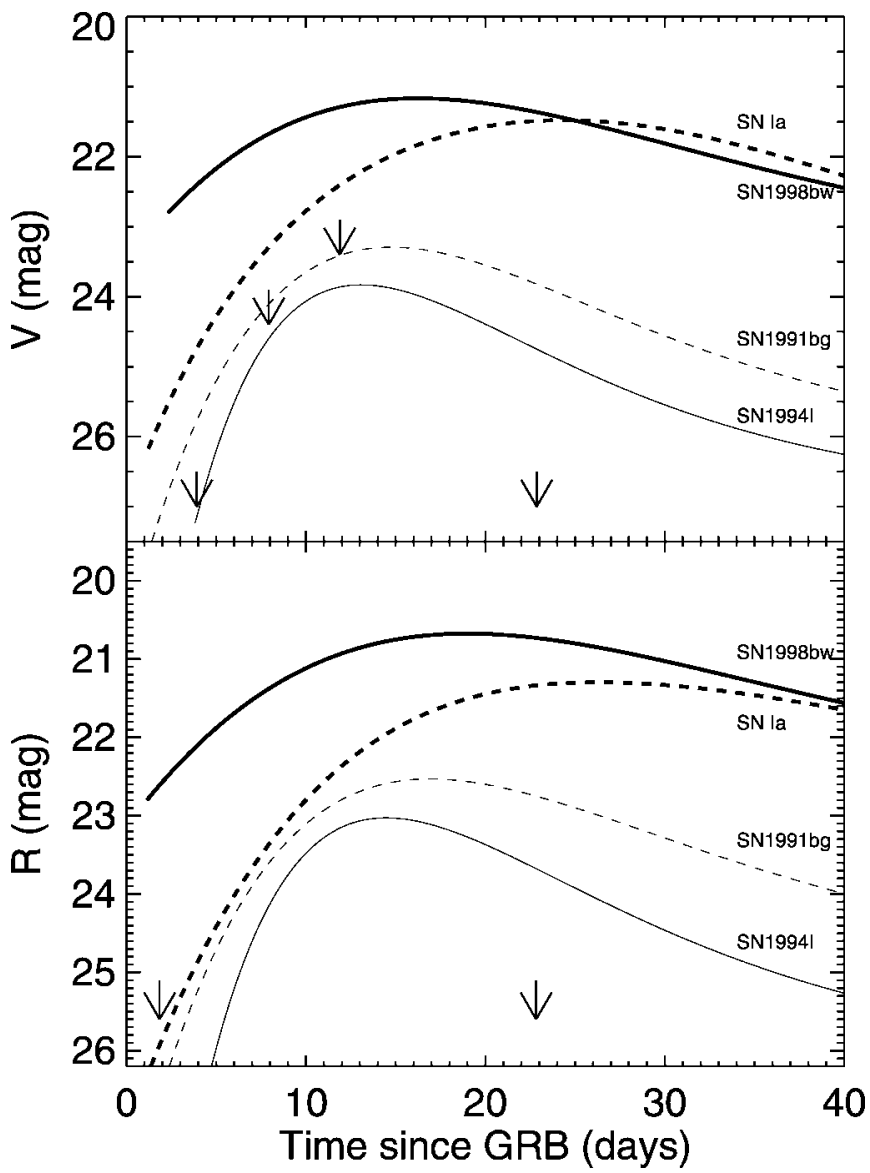

FIG. 2.-Upper limits (arrows) on variable sources (in the difference images) inside the GRB 050509B XRT error circle at the epochs given in Table 1 compared to the light curves of different SNe redshifted to $z=0.225$. Solid curves: Type Ic SNe. Dashed curves: Type Ia SNe. Thick solid curve: The hypernova SN 1998bw accompanying the long GRB 980425. Thin solid curve: The faint Ic supernova SN 1994I. Thick dashed curve: A typical Type Ia SN (stretch $=1)$. Thin dashed curve: A faint Type Ia SN similar to SN 1991bg. A Galactic extinction of $E(B-V)=0.019$ mag (Schlegel et al. 1998) toward GRB 050509B has been assumed.

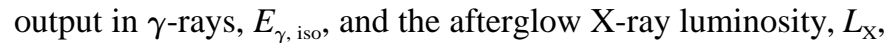
of GRB 050509B are significantly smaller than those of long GRBs. The most straightforward conclusion is that while GRB 050509B was a highly relativistic event (Gehrels et al. 2005), it was intrinsically less energetic compared to long GRBs, with relatively little energy $\left[\$ 10^{49}(\Omega / 4 \pi)\right.$ ergs] in highly relativistic ejecta with an initial Lorentz factor $\Gamma_{0} \gtrsim 100$ (from $E_{\gamma \text {, iso }}$ ) and with not much more energy in material with $\Gamma_{0} \gtrsim 3\left(E_{51} / n_{0}\right)^{1 / 8}$, where $E_{k \text {, iso }}=10^{51} E_{51}$ ergs is the isotropic equivalent energy in the afterglow shock and $n=n_{0} \mathrm{~cm}^{-3}$ is the external density. ${ }^{18}$ Moreover, the total observed energy from the burst was much smaller than the available energy in a NS-NS or NS-BH merger, or in most other progenitor models, suggesting that more energy was released in slower ejecta. The amount of energy in material above a certain initial four-velocity, $E\left(>\Gamma_{0} \beta_{0}\right)$, is very uncertain theoretically but may be constrained by our late-time upper limit on the optical emission.

\footnotetext{
${ }^{18}$ A higher $E_{k \text {, iso }}$ is possible for a very low external density. For $n \sim 10^{-6} \mathrm{~cm}^{-3}$, $E_{k, \text { iso }} \sim 10^{51}$ ergs for $z=0.225$ and $\sim 10^{52}-10^{53}$ ergs for $z \approx 3$ (Bloom et al. 2005; Lee et al. 2005). This would, however, require $E_{\gamma \text {, iso }} \ll E_{k \text {, iso }}$, i.e., a very inefficient prompt emission (compared to $E_{\gamma, \text { iso }} \gtrsim E_{k \text {, iso }}$ for long GRBs) and would not naturally reproduce the fact that $L_{\mathrm{X}} / E_{\gamma, \text { iso }}$ for GRB $050509 \mathrm{~B}$ is similar to that for long GRBs.
} 
There are two ways to produce the most readily detectable emission from the outflow associated with GRB 050509B. Either (1) it can originate in the shock created by the outflow as it drives into the ambient medium, similar to both a long GRB afterglow for relativistic ejecta and to a SN remnant for Newtonian ejecta, or (2) bright transient emission, dubbed a "miniSN" (Li \& Paczyński 1998; Rosswog \& Ramirez-Ruiz 2002), is produced by radioactive elements that are synthesized during the rapid decompression of very dense and neutron-rich material that is ejected during a NS-NS or a NS-BH merger (see, e.g., Rosswog et al. 1999). Our upper limit at $t \approx 22.8$ days constrains mainly the former mechanism, in particular the amount of energy in ejecta with $\Gamma_{0} \gtrsim 1.3\left(E_{51} / n_{0}\right)^{1 / 8}$, and suggests that the total energy in a relativistic outflow is significantly smaller in GRB 050509B than that in typical long GRBs. ${ }^{19}$ Our upper limit at $t=1.85$ days provides more stringent constraints on the latter mechanism, in which the emission is expected to peak around the optical-UV range within a day or so (up to a few days) with a semithermal spectrum (Li \& Paczyński 1998). The mini$\mathrm{SN}$ emission is mainly concentrated in a very narrow energy range (i.e., the optical) during (and near) the peak; therefore, the X-ray emission could have been easily missed by the Chandra observation of GRB $050509 \mathrm{~B}$ at $t \approx 2.5$ days.

Using the simplified model of Li \& Paczyński (1998), the optical flux from a mini-SN associated with GRB 050509B should have been a factor of $\sim 10^{3}\left(f / 10^{-3}\right)\left(M / 0.01 M_{\odot}\right)^{1 / 2}(3 v / \mathrm{c})^{1 / 2}$ higher than our upper limit at $t=1.85$ days, where $M$ and $v$ are the mass and velocity of the ejected material, and $f$ is the fraction of its rest energy that goes into radioactive decay of the elements with a decay timescale comparable to the time it takes the expanding ejecta to become optically thin, $t_{\tau}$. For a kinetic energy of $10^{51} E_{51}$ ergs, where $E_{51}=\left(M / 0.01 M_{\odot}\right)(3 v / c)^{2} \sim 1$, varying $M$ and $v$ within a reasonable range $(0.003 \lesssim$ $M / M_{\odot} \lesssim 1$ and $0.03 \lesssim v / c \lesssim 0.5$ ) would not change the optical luminosity by more than 1 order of magnitude. A larger uncertainty is the value of $f$, which reflects the amount of radioactive material synthesized in the accompanying NS-NS wind. From

\footnotetext{
${ }^{19}$ One possible caveat is the dependence of the afterglow brightness on the density of the burst environment (see Bloom et al. 2005); since the possible counterpart location on G1 spans a large range of densities, we have not folded this dependence in our conclusions.
}

the above simple arguments we derive an approximate upper limit of $f \leqq 10^{-5}$. We note here that the most efficient conversion of nuclear energy to the observable luminosity is provided by the elements with a decay timescale comparable to $t_{\tau}$. In reality, there is likely to be a large number of nuclides with a very broad range of decay timescales. Our limits thus place interesting constraints on the abundances and the lifetimes of the radioactive nuclides that form in the rapid decompression of nuclear-density matter: they should be either very short or very long when compared to $t_{\tau}$ so that radioactivity is inefficient in generating a large luminosity.

The above arguments suggest either that the intrinsic energy in the outflow from GRB 050509B was $\ll 10^{51} \mathrm{ergs}$, or alternatively, and arguably more likely, that it was close to the canonical value of $\sim 10^{51}$ ergs but most of this energy was in subrelativistic ejecta with a very small radioactive component during the optically thick expansion phase. ${ }^{20}$ The latter is very different from long/soft GRBs, which typically have $\sim 10^{51}$ ergs in highly relativistic ejecta with $\Gamma_{0} \gtrsim 100$. More short GRB afterglows are needed to establish whether the energetics of GRB 050509B is representative of the bulk of the short/hard GRB class.

Finally, our observations may place constraints on other possible models of short GRBs (e.g., Ramirez-Ruiz 2004; Tan et al. 2001; Rosswog et al. 2003, 2004; Shibata \& Sekiguchi 2003; Blackman \& Yi 1998; Davies et al. 2005). The strict optical upper limits derived in this Letter argue that these scenarios are only feasible if the transport of the energy is in the form of subrelativistic ejecta with little or almost no radioactivity, or in any other form of delayed energy input such as provided by a pulsar or by later mass ejection from a central source.

We thank Elena Pian and Alberto Castro-Tirado for comments and the ESO Paranal Science Operations staff, in particular Chris Lidman and Andreas O. Jaunsen, for efficiently conducting the observations reported here. The Dark Cosmology Centre is supported by the DNRF. The authors acknowledge benefits from collaboration within the EC FP5 Research Training Network "Gamma-Ray Bursts-An Enigma and a Tool."

\footnotetext{
${ }^{20}$ Such subrelativistic velocities could be the result of a significant entrainment of baryons into the outflow.
}

\section{REFERENCES}

Alard, C., \& Lupton, R. H. 1998, ApJ, 503, 325

Blackman, E. G., \& Yi, I. 1998, ApJ, 498, L31

Bloom, J. S., et al. 1999, Nature, 401, 453

2005, ApJ, submitted (astro-ph/0505480)

Castro-Tirado, A. J., et al. 2005, A\&A, 439, L15

Cenko, S. B., et al. 2005, GCN Circ. 3391, http://gcn.gsfc.nasa.gov/gen/gcn3/ 3391.gen3

Dado, S., Dar, A., \& De Rújula, A. 2005, GCN Circ. 3424, http://gcn.gsfc.nasa.gov/ $\mathrm{gcn} / \mathrm{gcn} 3 / 3424 . \mathrm{gcn} 3$

Dar, A., \& De Rújula, A. 2004, Phys. Rep., 405, 203

Davies, M. B., Levan, A. J., \& King, A. R. 2005, MNRAS, 356, 54

Fan, Y. Z., Zhang, B., Kobayashi, S., \& Mészáros, P. 2005, ApJ, 628, 867

Fynbo, J. P. U., et al. 2004, ApJ, 609, 962

Gal, R. R., et al. 2003, AJ, 125, 2064

Galama, T. J., et al. 1998, Nature, 395, 670

Garnavich, P. M., et al. 2003, ApJ, 582, 924

Gehrels, N., et al. 2004, ApJ, 611, 1005

. 2005, Nature, submitted (astro-ph/0505630)

Germany, L. M., et al. 2000, ApJ, 533, 320

Ghirlanda, G., Ghisellini, G., \& Celotti, A. 2004, A\&A, 422, L55

Gorosabel, J., et al. 2002, A\&A, 383, 112

Hjorth, J., et al. 2003, Nature, 423, 847

2005, GCN Circ. 3410, http://gcn.gsfc.nasa.gov/gcn/gcn3/3410.gcn3

Hurley, K., et al. 2002, ApJ, 567, 447
Kehoe, R., et al. 2001, ApJ, 554, L159

Klotz, A., Boër, M., \& Atteia, J.-L. 2003, A\&A, 404, 815

Kouveliotou, C., et al. 1993, ApJ, 413, L101

Lee, W. H., et al. 2005, ApJL, in press (astro-ph/0506104)

Li, L. X., \& Paczyński, B. 1998, ApJ, 507, L59

MacFadyen, A. I., \& Woosley, S. E. 1999, ApJ, 524, 262

Malesani, D., et al. 2004, ApJ, 609, L5

Nugent, P., Kim, A., \& Perlmutter, S. 2002, PASP, 114, 803

Ramirez-Ruiz, E. 2004, MNRAS, 349, L38

Richardson, D., et al. 2002, AJ, 123, 745

Richmond, M. W., et al. 1996, AJ, 111, 327

Rosswog, S., et al. 1999, A\&A, 341, 499

Rosswog, S., \& Ramirez-Ruiz, E. 2002, MNRAS, 336, L7

Rosswog, S., Ramirez-Ruiz, E., \& Davies, M. B. 2003, MNRAS, 345, 1077

Rosswog, S., Speith, R., \& Wynn, G. A. 2004, MNRAS, 351, 1121

Schlegel, D. J., Finkbeiner, D. P., \& Davis, M. 1998, ApJ, 500, 525

Shibata, M., \& Sekiguchi, Y. 2003, Phys. Rev. D, 68, 104020

Sodemann, M., \& Thomsen, B. 1994, A\&A, 292, 425

Soderberg, A. M., et al. 2005, ApJ, 627, 877

Stanek, K. Z., et al. 2003, ApJ, 591, L17

Tan, J. C., Matzner, C. D., \& McKee, C. F. 2001, ApJ, 551, 946

Yamazaki, R., Ioka, K., \& Nakamura, T. 2004, ApJ, 607, L103

Zeh, A., Klose, S., \& Hartmann, D. H. 2004, ApJ, 609, 952

Zhang, W., Woosley, S. E., \& MacFadyen, A. I. 2003, ApJ, 586, 356 